\title{
Chaos and Hopf Bifurcation Analysis of the Delayed Local Lengyel-Epstein System
}

\author{
Qingsong Liu, ${ }^{1}$ Yiping Lin, ${ }^{1}$ Jingnan Cao, ${ }^{2}$ and Jinde Cao ${ }^{3,4}$ \\ ${ }^{1}$ Department of Applied Mathematics, Kunming University of Science and Technology, Kunming, Yunnan 650500, China \\ ${ }^{2}$ School of Computer Science, Beijing University of Post Telecommunication, Beijing 100876, China \\ ${ }^{3}$ Department of Mathematics, Southeast University, Nanjing 210096, China \\ ${ }^{4}$ Department of Mathematics, Faculty of Science, King Abdulaziz University, Jeddah 21589, Saudi Arabia
}

Correspondence should be addressed to Yiping Lin; linyiping689@163.com

Received 4 January 2014; Accepted 25 February 2014; Published 30 March 2014

Academic Editor: Wenwu Yu

Copyright (C) 2014 Qingsong Liu et al. This is an open access article distributed under the Creative Commons Attribution License, which permits unrestricted use, distribution, and reproduction in any medium, provided the original work is properly cited.

\begin{abstract}
The local reaction-diffusion Lengyel-Epstein system with delay is investigated. By choosing $\tau$ as bifurcating parameter, we show that Hopf bifurcations occur when time delay crosses a critical value. Moreover, we derive the equation describing the flow on the center manifold; then we give the formula for determining the direction of the Hopf bifurcation and the stability of bifurcating periodic solutions. Finally, numerical simulations are performed to support the analytical results and the chaotic behaviors are observed.
\end{abstract}

\section{Introduction}

As is well known, in chemistry, the chlorite-iodide-malonic acid (CIMA) reaction is a typical example to indicate diffusion-driven instability mechanism. Castets et al. [1] discovered the formation of stationary three-dimensional structures of CIMA. Lengyel and Epstein [2,3] found that although there were five variables in the reaction, in fact, three of them in the reaction process were almost unchanged. Thus, it is able to simplify the original system to a two-dimensional model, which we call Lengyel-Epstein system. We know that the local system (the ODE model) of the Lengyel-Epstein system is taking the following form:

$$
\begin{gathered}
\dot{u}=a-u-4 \frac{u v}{1+u^{2}} \\
\dot{v}=\sigma b\left(u-\frac{u v}{1+u^{2}}\right),
\end{gathered}
$$

where, in the content of the CIMA reaction, $u$ and $v$ denote the chemical concentrations of the activator iodine $\left(\mathrm{I}^{-}\right)$and the inhibitor chlorite $\left(\mathrm{ClO}_{2}{ }^{-}\right)$, respectively, at time $t$. The positive parameters $a$ and $b$ are related to the feed concentration; similarly, the positive parameter $\sigma$ is a rescaling parameter depending on the concentration of the starch. Yi et al. [4] gave a detailed Hopf bifurcation analysis for this ODE model (and also the associated PDE model) by choosing $b$ as the bifurcation parameter and derived conditions on the parameters for determining the direction and the stability of the bifurcating periodic solution.

In order to reflect the dynamical behaviors of models depending on the past history of the system, it is often necessary to incorporate delays into the models. The ordinary and partial differential equations models involving time delays have been widely studied in fields as diverse as biology, population dynamics, neural networks, feedback controlled mechanical systems, machine tool vibrations, lasers, and economics [5-12]. While time delay effects can also be exploited to control nonlinear systems $[4,6,10,12-26]$, in [27], Çelik and Merdan considered the following delayed system:

$$
\begin{gathered}
\dot{u}=a-u-4 \frac{u v(t-\tau)}{1+u^{2}} \\
\dot{v}=\sigma b\left(u-\frac{u v(t-\tau)}{1+u^{2}}\right) .
\end{gathered}
$$

Using the delay parameter $\tau$ as a bifurcation parameter, they investigated the stability and Hopf bifurcation of the above system. 
Motivated by the above discussion, in the present paper, we devote our attention to the delayed local Lengyel-Epstein system taking the following form:

$$
\begin{gathered}
\dot{u}=a-u(t-\tau)-4 \frac{u(t-\tau) v(t-\tau)}{1+u^{2}(t-\tau)} \\
\dot{v}=\sigma b\left[u(t-\tau)-\frac{u(t-\tau) v(t-\tau)}{1+u^{2}(t-\tau)}\right],
\end{gathered}
$$

where $\tau$ is the positive time delay parameter. We consider the effect of time delay $\tau$ on $u$ and $v$ and give the conditions of the stability and the bifurcation of the positive equilibrium. By giving numerical simulations, we find that system (3) includes chaos.

This paper is organized as follows. In Section 2, we investigate the effect of the time delay $\tau$ on the stability of the positive equilibrium of system (3). In Section 3, we derive the direction and stability of Hopf bifurcation by using normal form and central manifold theory. Numerical simulations are carried out to illustrate the theoretical prediction and to explore the complex dynamics including chaos in Section 4. Section 5 summarizes the main conclusions.

\section{Stability Analysis and Hopf Bifurcation}

It is easy to see that system (3) has a unique positive equilibrium $E_{*}\left(u_{*}, v_{*}\right)$ with $u_{*}=\alpha, v_{*}=1+\alpha^{2}$ where $\alpha=a / 5$. as

Let $x=u-u_{*}, y=v-v_{*}$, and system (3) can be written

$$
\begin{aligned}
\dot{x}= & \left(\frac{3 \alpha^{2}-5}{1+\alpha^{2}}\right) x(t-\tau)+\left(\frac{-4 \alpha}{1+\alpha^{2}}\right) y(t-\tau) \\
& +f(x(t-\tau), y(t-\tau))+\text { h.o.t. } \\
\dot{y}= & \left(\frac{2 b \sigma \alpha^{2}}{1+\alpha^{2}}\right) x(t-\tau)+\left(\frac{-b \sigma \alpha}{1+\alpha^{2}}\right) y(t-\tau) \\
& +g(x(t-\tau), y(t-\tau))+\text { h.o.t., }
\end{aligned}
$$

where

$$
\begin{aligned}
& f(x(t-\tau), y(t-\tau)) \\
& =\frac{1}{2}\left[\frac{24 \alpha-8 \alpha^{2}}{\left(1+\alpha^{2}\right)^{2}} x^{2}(t-\tau)+\frac{-8+8 \alpha^{2}}{\left(1+\alpha^{2}\right)^{2}} x(t-\tau) y(t-\tau)\right] \\
& g(x(t-\tau), y(t-\tau)) \\
& =\frac{1}{2}\left[\frac{6 b \sigma \alpha-2 b \sigma \alpha^{3}}{\left(1+\alpha^{2}\right)^{2}} x^{2}(t-\tau)\right. \\
& \left.\quad+\frac{b \sigma \alpha^{3}-2 b \sigma}{\left(1+\alpha^{2}\right)^{2}} x(t-\tau) y(t-\tau)\right]
\end{aligned}
$$

and h.o.t denotes the higher order terms. Then, we obtain the linearized system

$$
\begin{aligned}
& \dot{x}=\left(\frac{3 \alpha^{2}-5}{1+\alpha^{2}}\right) x(t-\tau)+\left(\frac{-4 \alpha}{1+\alpha^{2}}\right) y(t-\tau) \\
& \dot{y}=\left(\frac{2 b \sigma \alpha^{2}}{1+\alpha^{2}}\right) x(t-\tau)+\left(\frac{-b \sigma \alpha}{1+\alpha^{2}}\right) y(t-\tau) .
\end{aligned}
$$

The corresponding characteristic equation is

$$
\lambda^{2}+2 M \lambda e^{-\lambda \tau}+N e^{-2 \lambda \tau}=0
$$

where $M=(1 / 2)\left(b \sigma \alpha /\left(1+\alpha^{2}\right)-\left(3 \alpha^{2}-5\right) /\left(1+\alpha^{2}\right)\right)>0$, $N=5 b \sigma \alpha /\left(1+\alpha^{2}\right)>0$ under the condition of

$$
b \sigma \alpha+5-3 \alpha^{2}>0
$$

For (7), we have the following Lemma.

Lemma 1. The two roots $\mu_{1,2}^{\prime}=-M \pm \sqrt{M^{2}-N}$ of (7) with $\tau=0$ have always negative parts if the condition $(H)$ holds.

The characteristic equation (7) can be rewritten as the following equation:

$$
\left(\lambda-\mu_{1}^{\prime} e^{-\lambda \tau}\right)\left(\lambda-\mu_{2}^{\prime} e^{-\lambda \tau}\right)=0
$$

Thus, $i \omega(\omega>0)$ is the root of (7) if and only if $\omega$ satisfies one of the following equations:

$$
i \omega-\mu_{1}^{\prime} e^{-i \omega \tau}=0 \quad \text { or } \quad i \omega-\mu_{2}^{\prime} e^{-i \omega \tau}=0 .
$$

If $M^{2}-N \geq 0$, then we have $\mu_{2}^{\prime} \leq \mu_{1}^{\prime}<0$ and it is easy to obtain

$$
\omega=-\mu_{1}^{\prime}, \quad \tau=\tau_{j}^{(1)}=-\frac{1}{\mu_{1}^{\prime}}\left(\frac{\pi}{2}+2 j \pi\right), \quad j=0,1,2, \ldots
$$

or

$$
\omega=-\mu_{2}^{\prime}, \quad \tau=\tau_{j}^{(2)}=-\frac{1}{\mu_{2}^{\prime}}\left(\frac{\pi}{2}+2 j \pi\right), \quad j=0,1,2, \ldots
$$

Noticing that $\mu_{2}^{\prime} \leq \mu_{1}^{\prime}<0$, therefore, we have $\tau_{j}^{(2)} \leq \tau_{j}^{(1)}$, $j=0,1,2, \ldots$

If $M^{2}-N<0$, then $\mu_{1}^{\prime}$ and $\mu_{2}^{\prime}$ are a pair of complex conjugate numbers and it is easy to get $\omega=\sqrt{N}$ and

$$
\tau=\tau_{j}^{(3)}=\frac{1}{\sqrt{N}}\left[\arccos \sqrt{1-\frac{M^{2}}{N}}+2 j \pi\right], \quad j=0,1,2, \ldots
$$

or

$$
\begin{aligned}
\tau & =\tau_{j}^{(4)} \\
& =\frac{1}{\sqrt{N}}\left[\arccos \sqrt{1-\frac{M^{2}}{N}}+(2 j+1) \pi\right], \quad j=0,1,2, \ldots
\end{aligned}
$$

Clearly, we have $\tau_{j}^{(3)}<\tau_{j}^{(4)}, j=0,1,2, \ldots$ 
Consequently, when $M^{2}-N \geq 0$, (7) with $\tau_{j}^{(k)}(k=1,2)$ has a pair of purely imaginary roots $\pm i \mu_{k}^{\prime}$; when $M^{2}-N<0$, (7) with $\tau_{j}^{(k)}(k=3,4)$ has a pair of purely imaginary roots $\pm i \sqrt{N}$

Summarizing the above and combining Lemma 1, we have the following result on the distribution of roots of (7).

\section{Lemma 2.}

(i) Assume that $M^{2}-N \geq 0$ and (H) hold; then when $\tau \in$ $\left[0, \tau_{0}^{(2)}\right)$, all roots of (7) have strictly negative real parts, while when $\tau=\tau_{0}^{(2)}$, (7) has a simple pair of purely imaginary roots $\pm i \mu_{2}^{\prime}$.

(ii) Assume that $M^{2}-N<0$ and (H) hold; then when $\tau \epsilon$ $\left[0, \tau_{0}^{(3)}\right)$, all roots of $(7)$ have strictly negative real parts, while when $\tau=\tau_{0}^{(3)}$, (7) has a simple pair of purely imaginary roots $\pm i \sqrt{N}$.

Let $\lambda(\tau)=\alpha(\tau) \pm i \omega(\tau)$ be the root of $(7)$ near $\tau=\tau_{j}^{(k)}(k=$ $1,2,3,4)$ satisfying $\alpha\left(\tau_{j}^{(k)}\right)=0, \omega\left(\tau_{j}^{(k)}\right)=-\mu_{k}^{\prime}(k=1,2)$, or $\omega\left(\tau_{j}^{(k)}\right)=\sqrt{N}(k=3,4 ; j=0,1,2, \ldots)$. It is not difficult to verify that the following result holds.

Lemma 3. If the condition $(H)$ holds, the transversality conditions $d \operatorname{Re} \lambda(\tau) /\left.d \tau\right|_{\tau=\tau_{j}^{(k)}} \neq 0(k=1,2,3,4 ; j=0,1,2, \ldots)$ hold.

From Lemma 3, we have the following result.

Lemma 4. If the condition $(H)$ holds, then when $M^{2}-N \geq 0$ (or $\left.M^{2}-N<0\right)$ and $\tau>\tau_{0}^{(2)}\left(\right.$ or $\left.\tau>\tau_{0}^{(3)}\right)$, (7) has at least one root with strictly positive real part.

By Lemmas 1-4, we have the following theorem.

Theorem 5. For system (3), assume that the condition $(H)$ holds; then the following statements are true.

(i) If $M^{2}-N \geq 0$ (or $M^{2}-N<0$ ), then the equilibrium $E_{*}\left(u_{*}, v_{*}\right)$ of system (3) is asymptotically stable for $\tau \in$ $\left[0, \tau_{0}^{(2)}\right)\left(\right.$ or $\left.\tau \in\left[0, \tau_{0}^{(3)}\right)\right)$.

(ii) If $M^{2}-N \geq 0$ (or $M^{2}-N<0$ ), then the equilibrium $E_{*}\left(u_{*}, v_{*}\right)$ of system (3) is unstable when $\tau>\tau_{0}^{(2)}$ (or $\left.\tau>\tau_{0}^{(3)}\right)$.

(iii) When $M^{2}-N \geq 0$ (or $\left.M^{2}-N<0\right), \tau=\tau_{j}^{(k)}(k=$ 1,2) $\left(\operatorname{or} \tau=\tau_{j}^{(k)}(k=3,4)\right)(j=0,1,2, \ldots)$ are Hopf bifurcation values of system (3).

\section{Direction and Stability of the Hopf Bifurcation}

In this section, using the method based on the normal form theory and center manifold theory introduced by Hassard et al. in [11], we study the direction of bifurcations and the stability of bifurcating periodic solutions. We denote the critical values $\tau_{j}^{(k)}$ as $\tau_{k}$; let $\tau=\tau_{k}+\mu$; then $\mu=0$ is the Hopf bifurcation value of system (3). Let $x=u-u_{*}, y=v-v_{*}$, $t=\bar{t} \tau$ and omit "." above $t$; then system (3) can be rewritten as

$$
\dot{U}(t)=\left(\tau_{k}+\mu\right)\left(B^{\prime} U(t)+C^{\prime} U(t-1)+F(x, y)\right),
$$

where

$$
\begin{aligned}
U(t) & =(x(t \tau), y(t \tau))^{T}, \\
B^{\prime}=\left(\begin{array}{ll}
0 & 0 \\
0 & 0
\end{array}\right), \quad C^{\prime} & =\left(\begin{array}{ll}
m_{1} & m_{2} \\
m_{3} & m_{4}
\end{array}\right), \quad F=\left(f_{1}, f_{2}\right)^{T},
\end{aligned}
$$

respectively. Let $\bar{x}(t)=x(t \tau), \bar{y}(t)=y(t \tau)$ and omit "." above $x$ and $y$; the nonlinear terms $f_{1}$ and $f_{2}$ are

$$
\begin{gathered}
m_{1}=\frac{3 \alpha^{2}-5}{1+\alpha^{2}}, \quad m_{2}=\frac{-4 \alpha}{1+\alpha^{2}}, \\
m_{3}=\frac{2 b \sigma \alpha^{2}}{1+\alpha^{2}}, \quad m_{4}=\frac{-b \sigma \alpha}{1+\alpha^{2}}, \\
f_{1}=\frac{1}{2}\left[A_{1} x^{2}(t-1)+A_{2} x(t-1) y(t-1)\right], \\
f_{2}=\frac{1}{2}\left[A_{3} x^{2}(t-1)+A_{4} x(t-1) y(t-1)\right], \\
A_{1}=\frac{24 \alpha-8 \alpha^{2}}{\left(1+\alpha^{2}\right)^{2}}, \quad A_{2}=\frac{-8+8 \alpha^{2}}{\left(1+\alpha^{2}\right)^{2}}, \\
A_{3}=\frac{6 b \sigma \alpha-2 b \sigma \alpha^{3}}{\left(1+\alpha^{2}\right)^{2}}, \quad A_{4}=\frac{b \sigma \alpha^{3}-2 b \sigma}{\left(1+\alpha^{2}\right)^{2}} .
\end{gathered}
$$

Define a family of operators as

$$
\begin{aligned}
L_{\mu} \varphi & =\left(\tau_{k}+\mu\right)\left[B^{\prime} \varphi(0)+C^{\prime} \varphi(-1)\right], \\
\varphi & =\left(\varphi_{1}, \varphi_{2}\right)^{T} \in C\left([-1,0], R^{2}\right) .
\end{aligned}
$$

By the Riesz representation theorem, there exists a matrix whose components are bounded variation functions $\eta(\theta, \mu)$ : $[-1,0] \rightarrow R^{2}$ such that

$$
L_{\mu} \varphi=\int_{-1}^{0} d \eta(\theta, \mu) \varphi(\theta) .
$$

In fact, choosing

$$
\eta(\theta, \mu)=B^{\prime} \delta(\theta)+C^{\prime} \delta(\theta+1),
$$

where

$$
\delta(\theta)= \begin{cases}1, & \theta=0 \\ 0, & \theta \neq 0\end{cases}
$$

is a Dirac delta function; then (17) is satisfied. 
For $\varphi=\left(\varphi_{1}, \varphi_{2}\right)^{T} \in C\left([-1,0], R^{2}\right)$, define

$$
\begin{gathered}
A(\mu) \varphi= \begin{cases}\frac{d \varphi(\theta)}{d \theta}, & \theta \in[-1,0), \\
\int_{-1}^{0} d \eta(s, \mu) \varphi(s), & \theta=0,\end{cases} \\
R(\mu) \varphi= \begin{cases}0, & \theta \in[-1,0), \\
h(\mu, \varphi), & \theta=0,\end{cases}
\end{gathered}
$$

where

$$
\begin{aligned}
h(\mu, \varphi) & =\left(\tau_{k}+\mu\right)\left(\begin{array}{l}
h_{1} \\
h_{2}
\end{array}\right), \quad \varphi=\left(\varphi_{1}, \varphi_{2}\right)^{T} \in C\left([-1,0], R^{2}\right), \\
h_{1} & =\frac{1}{2}\left[A_{1} \varphi_{1}^{2}(-1)+A_{2} \varphi_{1}(-1) \varphi_{2}(-1)\right], \\
h_{2} & =\frac{1}{2}\left[A_{3} \varphi_{1}^{2}(-1)+A_{4} \varphi_{1}(-1) \varphi_{2}(-1)\right] .
\end{aligned}
$$

Hence, (14) can be rewritten as

$$
\dot{U}_{t}=A(\mu) U_{t}+R(\mu) U_{t}
$$

where $U=(x(t), y(t))^{T}$ and $U_{t}(\theta)=U(t+\theta), \theta \in[-1,0]$. For $\psi \in C\left([0,1],\left(R^{2}\right)^{*}\right)$, define $A(0)=A$ and the adjoint operator $A^{*}$ of $A$ as

$$
A^{*} \psi(s)= \begin{cases}-\frac{d \psi(s)}{d s}, & s \in(0,1], \\ \int_{-1}^{0} d \eta^{T}(t, 0) \psi(-t), & s=0\end{cases}
$$

where $\eta^{T}$ is the transpose of the matrix $\eta$.

For $\varphi \in C\left([-1,0], R^{2}\right)$, and $\psi \in C\left([0,1],\left(R^{2}\right)^{*}\right)$, we define a bilinear inner product

$$
\begin{aligned}
\langle\psi(s), \varphi(\theta)\rangle= & \bar{\psi}(0) \varphi(0) \\
& -\int_{-1}^{0} \int_{\xi=0}^{\theta} \bar{\psi}(\xi-\theta) d \eta(\theta) \varphi(\xi) d \xi,
\end{aligned}
$$

where $\eta(\theta)=\eta(\theta, 0)$.

Since $\pm i \omega_{k} \tau_{k}$ are eigenvalues of $A$, they will also be the eigenvalues of $A^{*}$. The eigenvectors of $A$ and $A^{*}$ are calculated corresponding to the eigenvalues $+i \omega_{k} \tau_{k}$ and $-i \omega_{k} \tau_{k}$.

Lemma 6. $q(\theta)=(1, \rho)^{T} e^{i \omega_{k} \tau_{k} \theta}$ is the eigenvector of $A$ corresponding to $+i \omega_{k} \tau_{k} ; q^{*}(s)=(1 / K)(1, \delta)^{T} e^{i \omega_{k} \tau_{k} s}$ is the eigenvector of $A^{*}$ corresponding to $-i \omega_{k} \tau_{k}$ and

$$
\left\langle q^{*}(s), q(\theta)\right\rangle=1, \quad\left\langle q^{*}(s), \bar{q}(\theta)\right\rangle=0,
$$

where

$$
\begin{gathered}
\rho=\frac{i \omega_{k}-m_{1} e^{-i \omega_{k} \tau_{k}}}{m_{2} e^{-i \omega_{k} \tau_{k}}}, \quad \delta=\frac{-i \omega_{k}-m_{1} e^{i \omega_{k} \tau_{k}}}{m_{3} e^{i \omega_{k} \tau_{k}}}, \\
\bar{K}=1+\rho \bar{\delta}+\tau_{k}\left[m_{1}+m_{3} \bar{\delta}+\rho\left(m_{2}+m_{4} \bar{\delta}\right)\right] e^{-i \omega_{k} \tau_{k}}
\end{gathered}
$$

Following the algorithms explained in Hassard et al. [11], we can obtain the properties of Hopf bifurcation:

$$
\begin{gathered}
g_{20}=\frac{\tau_{k}}{\bar{K}}\left[A_{1} e^{-2 i \omega_{k} \tau_{k}}+A_{2} \rho e^{-2 i \omega_{k} \tau_{k}}\right. \\
\left.+\bar{\delta}\left(A_{3} e^{-2 i \omega_{k} \tau_{k}}+A_{4} \rho e^{-2 i \omega_{k} \tau_{k}}\right)\right], \\
g_{11}=\frac{\tau_{k}}{\bar{K}}\left[A_{1}+A_{2} \bar{\rho}+\bar{\delta}\left(A_{3}+A_{4} \bar{\rho}\right)\right], \\
g_{02}=\frac{\tau_{k}}{\bar{K}}\left[A_{1} e^{2 i \omega_{k} \tau_{k}}+A_{2} \bar{\rho} e^{2 i \omega_{k} \tau_{k}}\right. \\
\left.\quad+\bar{\delta}\left(A_{3} e^{2 i \omega_{k} \tau_{k}}+A_{4} \bar{\rho} e^{2 i \omega_{k} \tau_{k}}\right)\right], \\
g_{21}=\frac{\tau_{k}}{\bar{K}}\left\{A _ { 1 } \left(W_{11}^{(1)}(-1) e^{-i \omega_{k} \tau_{k}}+W_{20}^{(1)}(-1) e^{i \omega_{k} \tau_{k}}\right.\right. \\
\left.\quad+W_{11}^{(1)}(-1) e^{-i \omega_{k} \tau_{k}}\right)
\end{gathered}
$$

$$
\begin{gathered}
+A_{2}\left(W_{11}^{(2)}(-1) e^{-i \omega_{k} \tau_{k}}+\frac{1}{2} W_{20}^{(2)}(-1) e^{i \omega_{k} \tau_{k}}\right. \\
+\frac{1}{2} W_{20}^{(1)}(-1) \bar{\rho} e^{i \omega_{k} \tau_{k}} \\
\left.+W_{11}^{(1)}(-1) \rho e^{-i \omega_{k} \tau_{k}}\right) \\
+\bar{\delta}\left[A _ { 3 } \left(W_{11}^{(1)}(-1) e^{-i \omega_{k} \tau_{k}}+W_{20}^{(1)}(-1) e^{i \omega_{k} \tau_{k}}\right.\right. \\
\left.+W_{11}^{(1)}(-1) e^{-i \omega_{k} \tau_{k}}\right) \\
+A_{4}\left(W_{11}^{(2)}(-1) e^{-i \omega_{k} \tau_{k}}\right. \\
+\frac{1}{2} W_{20}^{(2)}(-1) e^{i \omega_{k} \tau_{k}} \\
+\frac{1}{2} W_{20}^{(1)}(-1) \bar{\rho} e^{i \omega_{k} \tau_{k}} \\
\left.\left.\left.+W_{11}^{(1)}(-1) \rho e^{-i \omega_{k} \tau_{k}}\right)\right]\right\}
\end{gathered}
$$

where

$$
\begin{aligned}
W_{20}(\theta)= & \frac{i g_{20}}{\omega_{k} \tau_{k}} q(0) e^{i \theta \omega_{k} \tau_{k}}+\frac{i \bar{g}_{02}}{3 \tau_{k} \omega_{k}} \bar{q}(0) e^{-i \theta \omega_{k} \tau_{k}} \\
& +R e^{2 i \theta \omega_{k} \tau_{k}}, \\
W_{11}(\theta)=- & \frac{i g_{11}}{\tau_{k} \omega_{k}} q(0) e^{i \theta \omega_{k} \tau_{k}}+\frac{i \bar{g}_{11}}{\tau_{k} \omega_{k}} \bar{q}(0) e^{-i \theta \omega_{k} \tau_{k}}+S .
\end{aligned}
$$




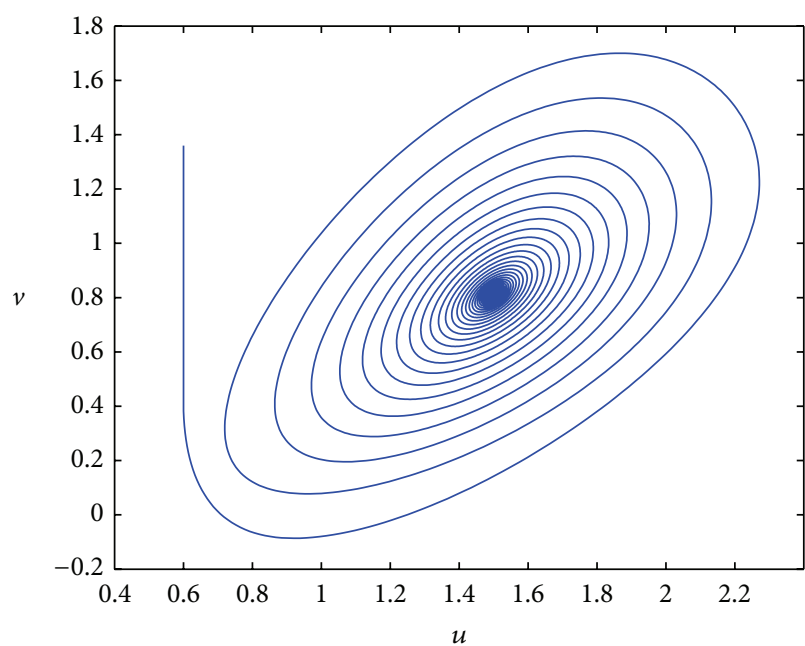

(a)

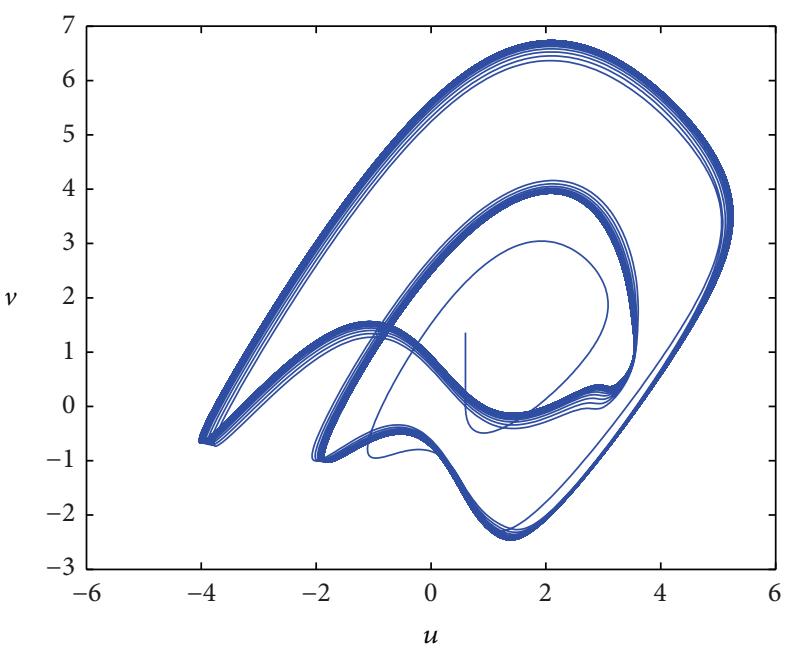

(c)

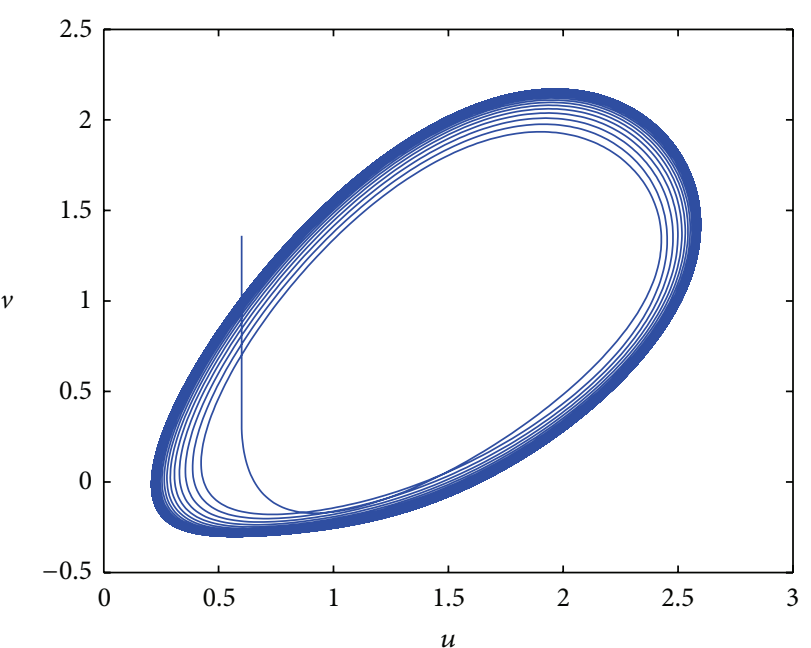

(b)



(d)

Figure 1: (a) $E_{*}$ is asymptotically stable equilibrium at $\tau=0.48$. (b) $E_{*}$ loses stability and stable periodic solution occurs at $\tau=0.79$. (c) A chaotic solution occurs at $\tau=3.6$. (d) The largest Lyapunov exponent diagram of system (32) for variable $\tau$.

We know that $R=\left(R^{(1)}, R^{(2)}\right) \in R^{2}$ and $S=\left(S^{(1)}, S^{(2)}\right) \in$ $R^{2}$ are constant vectors computed as

$$
\begin{gathered}
R=\left(\begin{array}{cc}
2 i \omega_{k}-m_{1} e^{-2 i \omega_{k} \tau_{k}} & -m_{2} e^{-2 i \omega_{k} \tau_{k}} \\
-m_{3} e^{-2 i \omega_{k} \tau_{k}} & 2 i \omega_{k}-m_{4} e^{-2 i \omega_{k} \tau_{k}}
\end{array}\right)^{-1} \\
\times\left(\begin{array}{c}
A_{1} e^{-2 i \omega_{k} \tau_{k}}+A_{2} \rho e^{-2 i \omega_{k} \tau_{k}} \\
A_{3} e^{-2 i \omega_{k} \tau_{k}}+A_{4} \rho e^{-2 i \omega_{k} \tau_{k}}
\end{array}\right) \\
S=\left(\begin{array}{ll}
m_{1} & m_{2} \\
m_{3} & m_{4}
\end{array}\right)^{-1}\left(\begin{array}{l}
A_{1}+A_{2} \bar{\rho} \\
A_{3}+A_{4} \bar{\rho}
\end{array}\right) .
\end{gathered}
$$

Thus, we can compute the following quantities:

$$
\begin{aligned}
c_{1}(0) & =\frac{i}{2 \omega_{k} \tau_{k}}\left(g_{20} g_{11}-2\left|g_{11}\right|^{2}-\frac{1}{3}\left|g_{02}\right|^{2}\right)+\frac{g_{21}}{2}, \\
\mu_{2} & =-\frac{\operatorname{Re}\left\{c_{1}(0)\right\}}{\operatorname{Re}\left\{\lambda^{\prime}\left(\tau_{k}\right)\right\}},
\end{aligned}
$$

$$
\begin{aligned}
& \beta_{2}=2 \operatorname{Re}\left\{c_{1}(0)\right\} \\
& T_{2}=-\frac{\operatorname{Im}\left\{c_{1}(0)\right\}+\mu_{2} \operatorname{Im}\left\{\lambda^{\prime}\left(\tau_{k}\right)\right\}}{\omega_{k} \tau_{k}} .
\end{aligned}
$$

These expressions give a description of the bifurcating periodic solutions in the center manifold of system (3) at critical values $\tau=\tau_{k}$ and when $\operatorname{Re}\left\{\lambda^{\prime}\left(\tau_{k}\right)\right\}>0$ which can be stated as follows:

(i) $\mu_{2}$ gives the direction of Hopf bifurcation; if $\mu_{2}>$ $0\left(\mu_{2}<0\right)$, the Hopf bifurcation is supercritical (subcritical).

(ii) $\beta_{2}$ determines the stability of bifurcating periodic solution; the periodic solution is stable (unstable) if $\beta_{2}<0\left(\beta_{2}>0\right)$. 
(iii) $T_{2}$ denotes the period of bifurcating period solutions; if $T_{2}>0\left(T_{2}<0\right)$, the period increases (decreases).

\section{Numerical Simulations}

To demonstrate the algorithm for determining the existence of Hopf bifurcation in Section 2 and the direction and stability of Hopf bifurcation in Section 3, we carry out numerical simulations on a particular case of (3) in the following form:

$$
\begin{aligned}
& \dot{u}=3-u(t-\tau)-4 \frac{u(t-\tau) v(t-\tau)}{1+u^{2}(t-\tau)} \\
& \dot{v}=0.8 \times 2\left[u(t-\tau)-\frac{u(t-\tau) v(t-\tau)}{1+u^{2}(t-\tau)}\right],
\end{aligned}
$$

where $a=3, b=2$, and $\sigma=0.8$. It is easy to show that system (32) has unique positive equilibrium $E_{*}(0.6,1.36)$, $b \sigma \alpha+5-3 \alpha^{2}=4.88>0$ and $M^{2}-N=-0.3106<0$. From the discussion of Section 2, we have $\omega_{0}=\sqrt{N}=1.8787$; by calculation, we obtain $\tau_{0}^{(3)}=0.6758$.

We can see from Figure 1(a) that $E_{*}$ is asymptotically stable at $\tau=0.48<\tau_{0}^{(3)}=0.6758$, while $E_{*}$ loses stability and Hopf bifurcation occurs when $\tau>\tau_{0}^{(3)}$; see Figure 1(b) at $\tau=0.79>\tau_{0}^{(3)}$. Using the algorithm derived in Section 3, we obtain that $\mu_{2}=2.134, \beta_{2}=-0.857$, and $T_{2}=2.128$, and we know that the Hopf bifurcation is supercritical, bifurcating periodic solutions are stable, and periods increase, whereas with parameter $\tau$ increasing chaotic solution occurs; see Figure 1(c) for $\tau=3.6>\tau_{0}^{(3)}$. In Figure 1(d), largest Lyapunov exponent diagram is plotted for variable $\tau$. It is easy to know when $\tau>3.5$; the Lyapunov exponent is almost positive; then the chaotic solutions occur.

\section{Conclusions}

In this paper, we investigate the effect of the time delay $\tau$ on the stability of the positive equilibrium of the delayed local Lengyel-Epstein system and derive the direction and stability of Hopf bifurcation. Numerical simulations are carried out to illustrate the theoretical prediction and to explore the complex dynamics including chaos.

\section{Conflict of Interests}

The authors declare that there is no conflict of interests regarding the publication of this paper.

\section{Acknowledgments}

The authors are grateful to the reviewers for their valuable comments and suggestions which have led to an improvement of this paper. This research is supported by the National Natural Science Foundation of China (Grant no. 11061016).

\section{References}

[1] V. Castets, E. Dulos, J. Boissonade, and P. de Kepper, "Experimental evidence of a sustained standing Turing-type nonequilibrium chemical pattern," Physical Review Letters, vol. 64, no. 24, pp. 2953-2956, 1990.

[2] I. Lengyel and I. R. Epstein, "Modeling of turing structures in the chlorite-iodide-malonic acid-starch reaction system," Science, vol. 251, no. 4994, pp. 650-652, 1991.

[3] I. Lengyel and I. R. Epstein, "A chemical approach to designing Turing patterns in reaction-diffusion systems," Proceedings of the National Academy of Sciences of the United States of America, vol. 89, no. 9, pp. 3977-3979, 1992.

[4] F. Yi, J. Wei, and J. Shi, "Diffusion-driven instability and bifurcation in the Lengyel-Epstein system," Nonlinear Analysis: Real World Applications, vol. 9, no. 3, pp. 1038-1051, 2008.

[5] L. J. S. Allen, An Introduction to Mathematical Biology, Prentice Hall, Upper Saddle River, NJ, USA, 2007.

[6] B. Balachandran, T. Katlmar-Nagy, and D. E. Gilsinn, Delay Differential Equations: Recent Advances and New Directions, Springer, New York, NY, USA, 2009.

[7] R. Bellman and K. L. Cooke, Differential-Difference Equations, Academic Press, New York, NY, USA, 1963.

[8] I. R. Epstein and J. A. Pojman, An Introduction to Nonlinear Chemical Dynamics, Oxford University Press, Oxford, UK, 1998.

[9] J. Hale, Theory of Functional Differential Equations, Springer, New York, NY, USA, 2nd edition, 1977.

[10] Y. Kuang, Delay Differential Equations with Applications in Population Dynamics, vol. 191 of Mathematics in Science and Engineering, Academic Press, Boston, Mass, USA, 1993.

[11] B. D. Hassard, N. D. Kazarinoff, and Y. H. Wan, Theory and Applications of Hopf Bifurcation, vol. 41 of London Mathematical Society Lecture Note Series, Cambridge University Press, Cambridge, Mass, USA, 1981.

[12] J. D. Murray, Mathematical Biology. I, vol. 17 of Interdisciplinary Applied Mathematics, Springer, New York, NY, USA, 3rd edition, 2002.

[13] H. Akkocaoğlu, H. Merdan, and C. Çelik, "Hopf bifurcation analysis of a general non-linear differential equation with delay," Journal of Computational and Applied Mathematics, vol. 237, no. 1, pp. 565-575, 2013.

[14] J. Bélair and S. A. Campbell, "Stability and bifurcations of equilibria in a multiple-delayed differential equation," SIAM Journal on Applied Mathematics, vol. 54, no. 5, pp. 1402-1424, 1994.

[15] K. L. Cooke and Z. Grossman, "Discrete delay, distributed delay and stability switches," Journal of Mathematical Analysis and Applications, vol. 86, no. 2, pp. 592-627, 1982.

[16] C. Çelik, "Hopf bifurcation of a ratio-dependent predator-prey system with time delay," Chaos, Solitons \& Fractals, vol. 42, no. 3, pp. 1474-1484, 2009.

[17] K. Li and J. Wei, "Stability and Hopf bifurcation analysis of a prey-predator system with two delays," Chaos, Solitons \& Fractals, vol. 42, no. 5, pp. 2606-2613, 2009.

[18] X. Li, S. Ruan, and J. Wei, "Stability and bifurcation in delaydifferential equations with two delays," Journal of Mathematical Analysis and Applications, vol. 236, no. 2, pp. 254-280, 1999.

[19] S. Ruan and J. Wei, "On the zeros of transcendental functions with applications to stability of delay differential equations with two delays," Dynamics of Continuous, Discrete \& Impulsive Systems. A. Mathematical Analysis, vol. 10, no. 6, pp. 863-874, 2003. 
[20] X.-P. Yan and Y.-D. Chu, "Stability and bifurcation analysis for a delayed Lotka-Volterra predator-prey system," Journal of Computational and Applied Mathematics, vol. 196, no. 1, pp. 198210, 2006

[21] J. Wei and S. Ruan, "Stability and bifurcation in a neural network model with two delays," Physica D, vol. 130, no. 3-4, pp. 255-272, 1999.

[22] M. Xiao, W. X. Zheng, and J. Cao, "Hopf bifurcation of an $(\mathrm{n}+1)$-neuron BAM neural network model with delays," IEEE Transactions on Neural Networks and Learning Systems, vol. 24, pp. 118-132, 2013.

[23] J. Cao and M. Xiao, "Stability and Hopf bifurcation in a simplified BAM neural network with two time delays," IEEE Transactions on Neural Networks, vol. 18, no. 2, pp. 416-430, 2007.

[24] W. He and J. Cao, "Stability and bifurcation of a class of discretetime neural networks," Applied Mathematical Modelling, vol. 31, no. 10, pp. 2111-2122, 2007.

[25] J. Cao and Y. Wan, "Matrix measure strategies for stability and synchronization of inertial BAM neural network with time delays," Neural Networks, vol. 53, pp. 165-172, 2014.

[26] X. Yang, J. Cao, and Z. Yang, "Synchronization of coupled reaction-diffusion neural networks with time-varying delays via pinning-impulsive controller," SIAM Journal on Control and Optimization, vol. 51, no. 5, pp. 3486-3510, 2013.

[27] C. Çelik and H. Merdan, "Hopf bifurcation analysis of a system of coupled delayed-differential equations," Applied Mathematics and Computation, vol. 219, no. 12, pp. 6605-6617, 2013. 


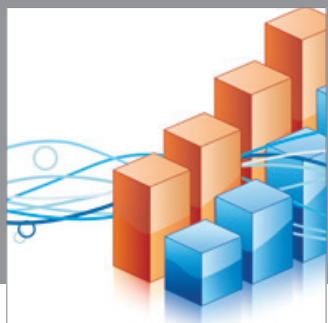

Advances in

Operations Research

mansans

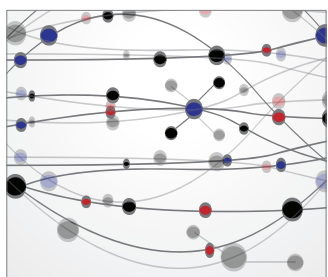

The Scientific World Journal
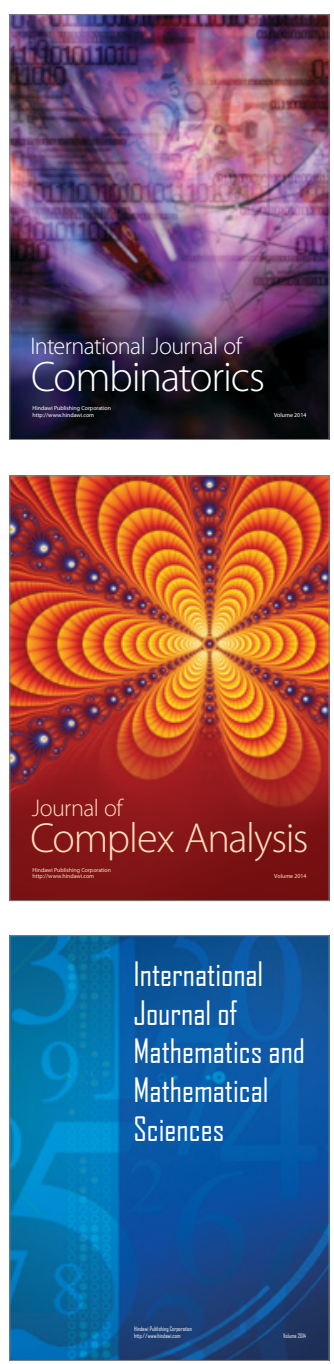
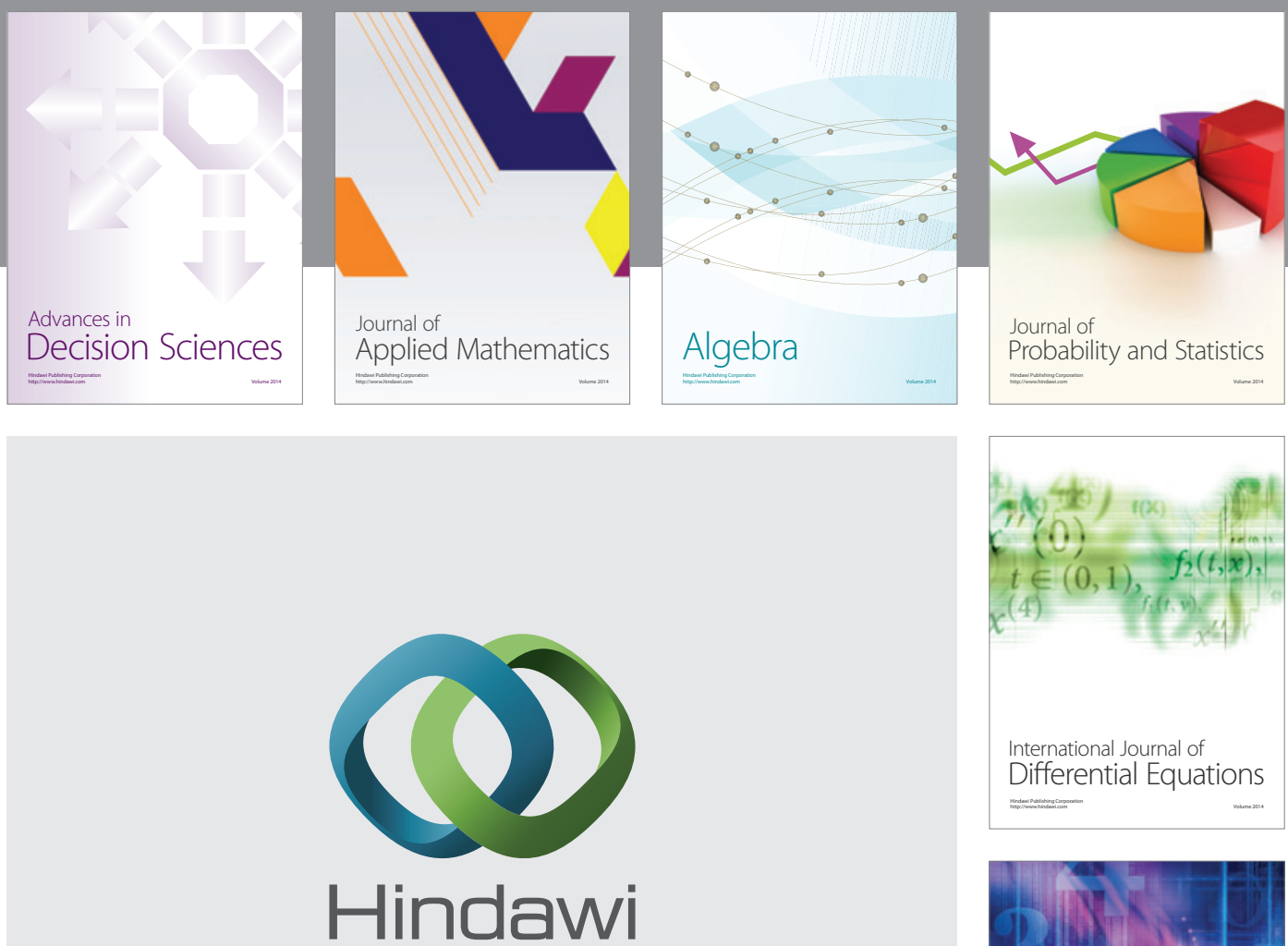

Submit your manuscripts at http://www.hindawi.com
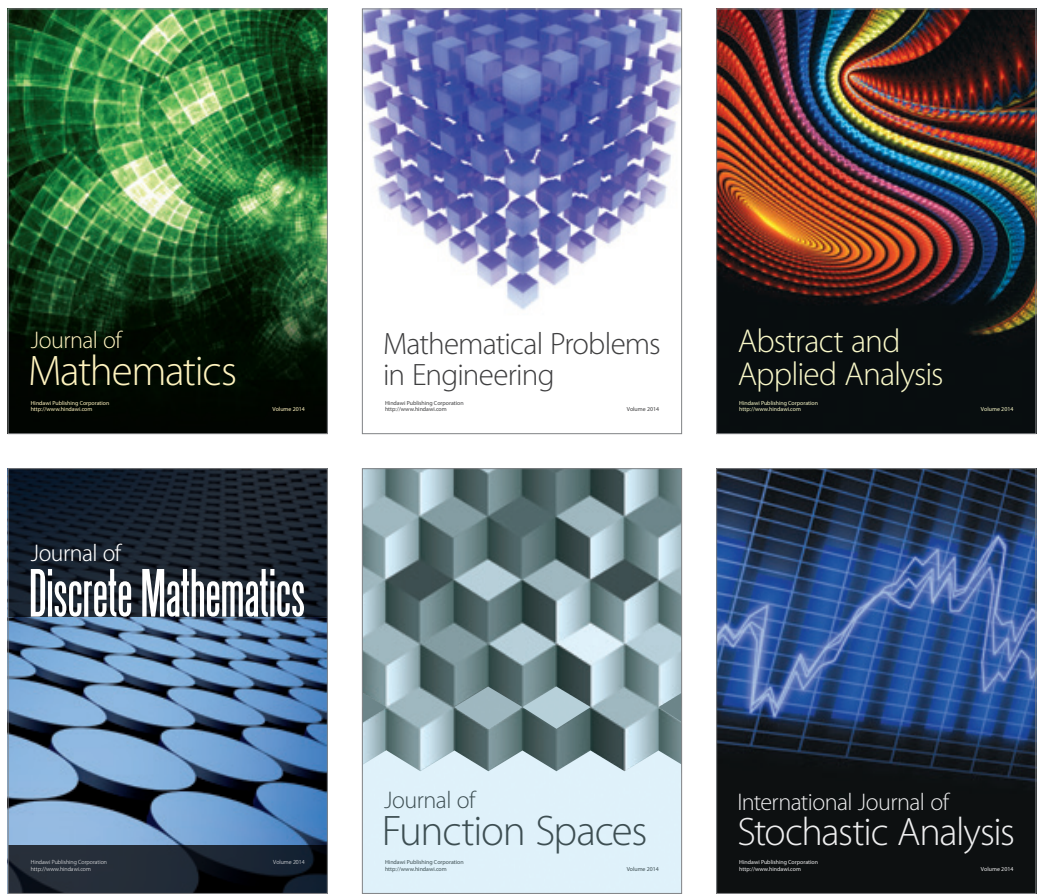

Journal of

Function Spaces



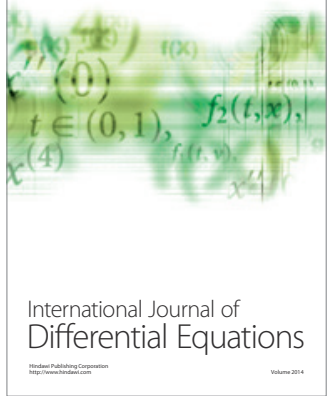
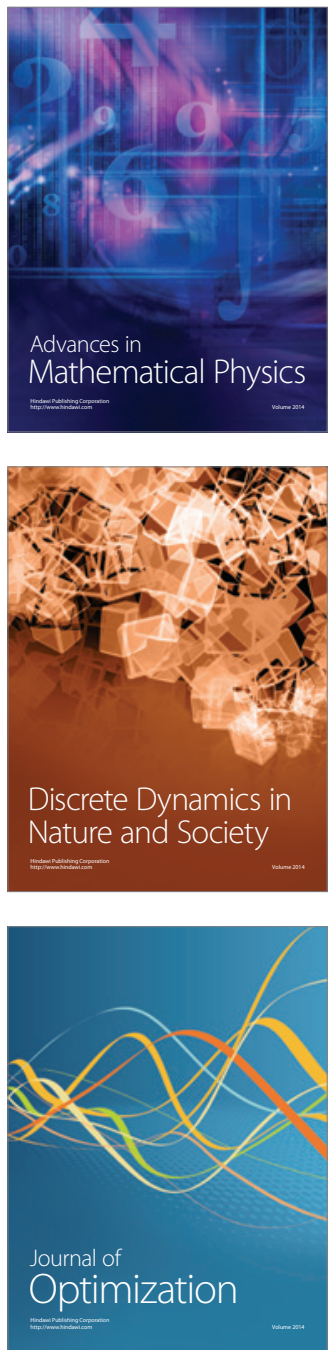